\title{
Case Series \\ A Novel Revision to the Classical Transnasal Topical Sphenopalatine Ganglion Block for the Treatment of Headache and Facial Pain
}

Kenneth D. Candido, MD ${ }^{1,2}$, Scott T. Massey, MD1, Ruben Sauer, MD1, Raheleh Rahimi Darabad, MD', and Nebojsa Nick Knezevic, MD, PhD ${ }^{1,2}$

From: ${ }^{1}$ Department of Anesthesiology, Advocate Illinois Masonic Medical Center, Chicago, IL; and 'Department of Anesthesiology, University of Illinois, Chicago, IL

Additional Author Affiliation information on pp. E776-E777.

Address Correspondence: Kenneth D. Candido, MD

Chairman and Professor Department of Anesthesiology Advocate Illinois Masonic Medical Center 836 W. Wellington Ave. Suite 4815 Chicago, IL 60657 E-mail:kdcandido@yahoo.com

Disclaimer: There was no external funding in the preparation of this manuscript. Conflict of interest: Each author certifies that he or she, or a member of his or her immediate family, has no commercial association (i.e., consultancies, stock ownership, equity interest, patent/licensing arrangements, etc.) that might post a conflict of interest in connection with the submitted manuscript.

Manuscript received: 06-13-2013 Revised manuscript received: 07-26-2013 Accepted for publication: 08-07-2013

Free full manuscript: www.painphysicianjournal.com
Background: The sphenopalatine ganglion (SPG) is located with some degree of variability near the tail or posterior aspect of the middle nasal turbinate. The SPG has been implicated as a strategic target in the treatment of various headache and facial pain conditions, some of which are featured in this manuscript. Interventions for blocking the SPG range from minimally to highly invasive procedures often associated with great cost and unfavorable risk profiles.

Objective: The purpose of this pilot study was to present a novel, FDA-cleared medication delivery device, the Tx360® nasal applicator, incorporating a transnasal needleless topical approach for SPG blocks. This study features the technical aspects of this new device and presents some limited clinical experience observed in a small series of head and face pain cases.

Study Design: Case series.

Settings: Pain management center, part of teaching-community hospital, major metropolitan city, United States.

Methods: After Institutional Review Board (IRB) approval, the technical aspects of this technique were examined on 3 patients presenting with various head and face pain conditions including trigeminal neuralgia (TN), chronic migraine headache (CM), and post-herpetic neuralgia (PHN).

The subsequent response to treatment and quality of life was quantified using the following tools: the 11-point Numeric Rating Scale (NRS), Modified Brief Pain Inventory - short form (MBPI-sf), Patient Global Impression of Change (PGIC), and patient satisfaction surveys. The Tx360® nasal applicator was used to deliver $0.5 \mathrm{~mL}$ of ropivacaine $0.5 \%$ and $2 \mathrm{mg}$ of dexamethasone for SPG block. Post-procedural assessments were repeated at 15 and 30 minutes, and on days one, 7, 14, and 21 with a final assessment at 28 days post-treatment. All patients were followed for one year. Individual patients received up to 10 SPG blocks, as clinically indicated, after the initial 28 days.

Results: Three women, ages 43,18 , and 15, presented with a variety of headache and face pain disorders including TN, CM, and PHN. All patients reported significant pain relief within the first 15 minutes post-treatment. A high degree of pain relief was sustained throughout the 28 day follow-up period for 2 of the 3 study participants. All 3 patients reported a high degree of satisfaction with this procedure. One patient developed minimal bleeding from the nose immediately post-treatment which resolved spontaneously in less than 5 minutes. Longer term follow-up (up to one year) demonstrated that additional SPG blocks over time provided a higher degree and longer lasting pain relief.

Limitations: Controlled double blind studies with a higher number of patients are needed to prove efficacy of this minimally invasive technique for SPG block.

Conclusion: SPG block with the $T \times 360 \circledR$ is a rapid, safe, easy, and reliable technique to accurately deliver topical transnasal analgesics to the area of mucosa associated with the SPG. This intervention can be delivered in as little as 10 seconds with the novice provider developing proficiency very quickly. Further investigation is certainly warranted related to technique efficacy, especially studies comparing efficacy of Tx360 and standard cotton swab techniques.

Key words: Trigeminal neuralgia, trigeminal neuropathy, migraine, headache, post-herpetic neuralgia, sphenopalatine ganglion block

Pain Physician 2013; 16:E769-E778 
T here are a wide variety of techniques described in the literature for managing head and face pain incorporating sphenopalatine ganglion (SPG) blocks. Unfortunately, there has been a paucity of evidence-based research conducted on these therapeutic modalities. Interventions range from non-invasive to significantly invasive and from cumbersome, technical, and expensive to fast, simple, and inexpensive (1).

Among the variety of SPG-targeted interventions examined, the subzygomal approach ensures more accurate delivery of the chosen agent and is likely more reliable than traditional transnasal topical approaches $(2,3)$. Despite the accuracy, this approach is significantly more invasive, painful, and expensive, and is associated with much greater demands of time and technical proficiency.

A transnasal SPG block, wherein the SPG area is accessed via the naris (4-6) is the simplest, safest, and least expensive of the SPG interventions. With this technique, a topical anesthetic blocking agent can be delivered with great accuracy to the area of mucosa associated with the SPG $(7,8)$. Although several modifications to the basic technique have been reported, the foundation for these procedures remains the same: to deliver topical anesthetic to the SPG. Until recently, the basic technique has essentially remained unchanged for over a century, and was popularized by Dr. John Bonica in his classic text, Management of Pain, first published in 1953 (9). A novel device, the $\mathrm{T} \times 360^{\oplus}$, overcomes certain inaccuracy and patient comfort issues associated with the historically used pledget-requiring and cotton-tipped swab transnasal techniques. In addition, the design of this device allows for its use with deviated septum patients in contrast to traditional techniques. This device is designed to deliver the anesthetic more precisely to the discrete area of mucosa associated with the SPG.

The purpose of this preliminary report was to demonstrate the technical aspects and ease of use of the Tx360 to facilitate SPG blocks. We believe this manuscript provides an overview of a novel technique that serves to overcome some traditional limitations in this approach. There is certainly an unmet need to improve transnasal techniques for SPG block, and we propose the Tx360 may play an important future role in improving on this considerably long-practiced, minimally invasive technique.

\section{Methods}

The protocol was approved by the Advocate
Healthcare Internal Review Board, and the study was registered with clinicaltrials.gov. Selected patients who had been recommended SPG block for their headache or facial pain syndromes were offered the Tx360 nasal applicator device as one option to block the SPG. All patients had failed other conservative approaches, including forehead blocks (supraorbital; supratrochlear nerves) and trigger point injections; medication management; biofeedback; and relaxation techniques. After informed consent was obtained, all patients received the same medication and the same dosage consisting of $0.5 \mathrm{~mL}$ of $0.5 \%$ ropivacaine and $2 \mathrm{mg}$ of dexamethasone sodium phosphate delivered transnasally and bilaterally (total of $1 \mathrm{~mL}$ per side) via the Tx360 . In addition to routine history and physical exam, certain information was collected prior to performing the procedure. Pain intensity was recorded based on the 11-point Numeric Rating Scale (NRS). The Modified Brief Pain Inventory-short form (MBPI-sf) was completed and a baseline was established. The MBPI-sf measures the degree of pain interference with common daily activities on $0-10$ scale, with 0 representing "no interference" and 10 representing "complete interference." At 15 minutes post-treatment, the NRS score was reassessed. At 30 minutes, the NRS score and the Patient's Global Impression of Change (PGIC) were recorded. On follow-up days one, 7, 14, 21, and 28, NRS scores, PGIC data, and use of medication were also recorded. We continued following these patients for up to one year, and they were allowed to have as many injections as needed after the initial 28 day follow-up. No individual patient received more than 10 procedures over the one year observation period until completion of the study. The heterogeneous patient sample was intended to feature a variety of applications.

\section{Procedure}

The technique proved to be very easy to learn with a high degree of proficiency rapidly achieved. Training included the assistance of an anatomically correct model to observe the device mechanics within the nasal cavity. A $1 \mathrm{~mL}$ luer-lock syringe pre-loaded with anesthetic fits easily into the Tx360 chamber (Fig. 1). Once the device is introduced into the naris and the syringe is advanced into the barrel of the device, a soft, thin, flexible, atraumatic, curved catheter advances posterior to clear the inferior turbinate. The catheter tip sits up in a position medial, inferior, and posterior to the target mucosa. The opening of the catheter tip is designed to specifically direct the spray of anesthetic in a superior, 


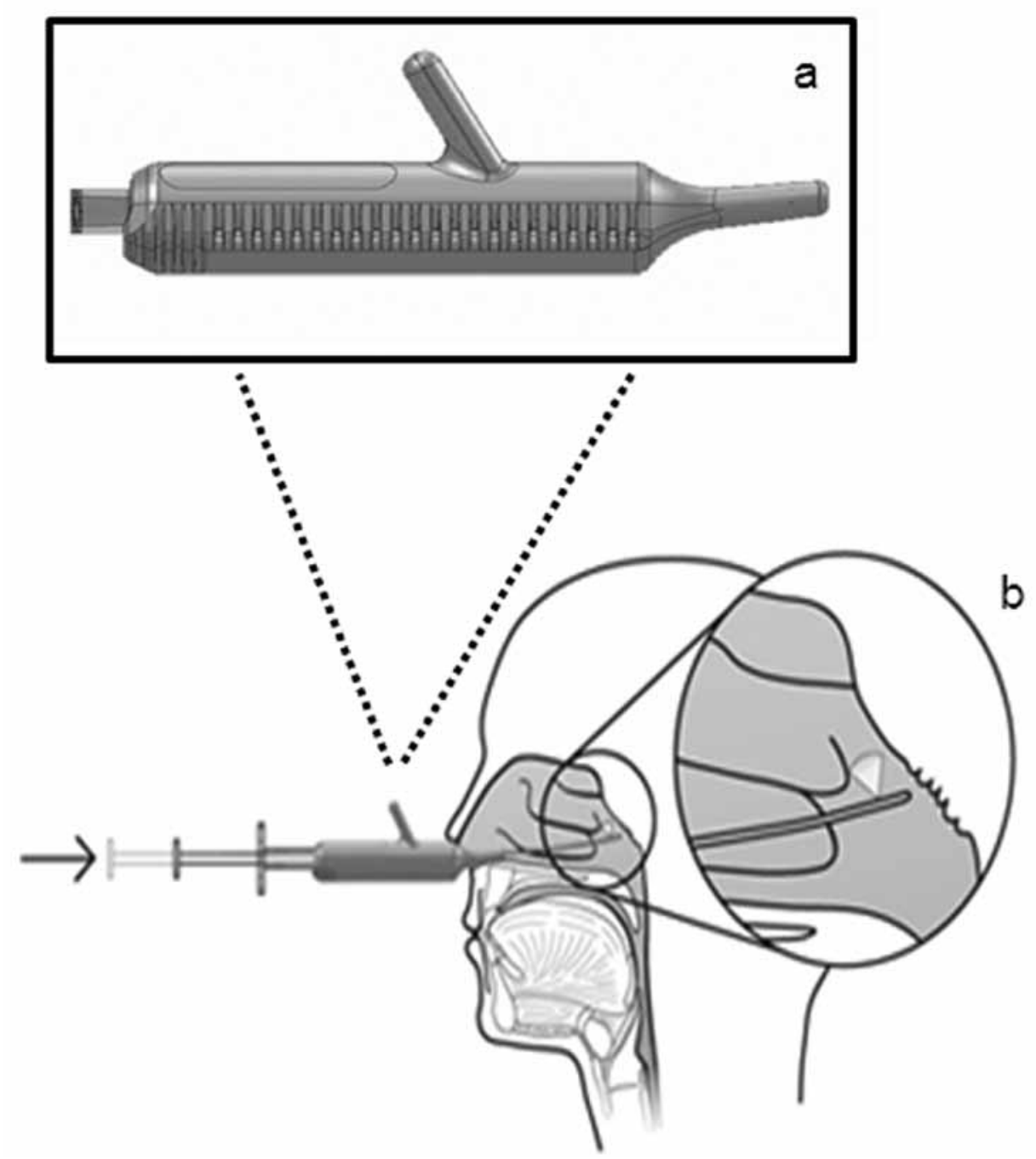

Fig. 1. Schematic use of the Tx360 ${ }^{\circledR}$ device (a) and expanded view of the nasal cavity depicting the boundary between the nasal cavity and the SPG (b).

lateral, and anterior direction with the width of spray diffusion wide enough to account for anatomic variability of the sphenopalatine foramen (SPF). The procedure takes as little as 10 seconds to perform in unsedated patients who may remain seated for its performance.

\section{Results}

\section{Patient \# 1}

A 43-year-old woman presented with paroxysmal attacks of sharp, shooting pain in the left cheek, each lasting less than one minute. Her pain was exacerbated by exposure to cold and wind. These brief attacks oc- curred approximately every $2-3$ hours, followed by numbness and dull pain, intensity $10 / 10$, lasting about 45 minutes. This problem lasted for 18 months. A dental consult resulted in a tooth extraction and root canal which did not provide pain relief. Subsequently, the patient was seen by 2 neurologists and diagnosed with trigeminal neuralgia (TN) based on the International Headache Classification (ICHD-II) (10) before being referred to our pain clinic. She underwent pharmacotherapy with carbamazepine, ibuprofen, pregabalin, tramadol, and hydrocodone/acetaminophen which provided no relief for acute flares. Before SPG block with the Tx360, pre-procedural pain level was rated 
$8 / 10$, which decreased to $1 / 10$ by the minute 15 reassessment, ultimately decreasing to $0 / 10$ through the Day 7 follow-up (Fig. 2). Between Day 14 and Day 21, pain levels trended upwards to 4/10. Her PGIC was "very much improved" by Day 7, and subsequently trended downwards to "much improved" through the Day 28 follow-up. Satisfaction scores were 4 and 5 out of 5 during the first 28 days. Pre-treatment interference were $8-8 / 10$ in 6 of 7 categories. Subsequent follow-ups demonstrated complete resolution of interference with only a relatively small degree of interference noted at the Day 28 follow-up. The patient developed minimal bleeding from the nose on the first day which stopped spontaneously in < 15 minutes. No other complications were observed. We followed-up the patient for one year, after which she stated that she was comfortable and required no additional analgesic therapy of any kind. She received 2 additional SPG blocks with longer pain relief than the initial block and without additional episodes of nasal bleeding.

\section{Patient \# 2}

An 18-year-old woman was referred from Children's Memorial Hospital for evaluation of her refrac- tory migraine headaches. Her initial episode was secondary to a fall in October 2010 during cheerleading practice. The patient fell on her left head/neck/shoulder area. Ten days later, she began experiencing migraine headaches with the most intense pain being in the left infraorbital region and right supraorbital region. Since that time, she had been afflicted with chronic refractory migraine exacerbations. Infraorbital and supraorbital nerve blocks were administered which provided some degree of relief for $3-5$ weeks at a time, and trigger point injections of the trapezius muscles were not helpful. The patient denied changes in vision, hearing, smell, or speech and denies extremity paresthesia. Pharmacotherapy included dihydroergotamine, topiramate, and escitalopram which offered marginal temporary pain relief down to a level of 5/10 on the NRS pain scale. Physical examination revealed no neurologic or cognitive deficit. Following a single SPGB, the patient experienced sustained pain relief over the 28-day posttreatment period. Her pain NRS score remained at 2/10 for the 28-day period (Fig. 2), the PGIC was "very much improved" the first week, and her satisfaction scores were between $4 / 5$ and 5/5. We followed-up the patient for one year. She received 4 more SPGBs, achieving lon-

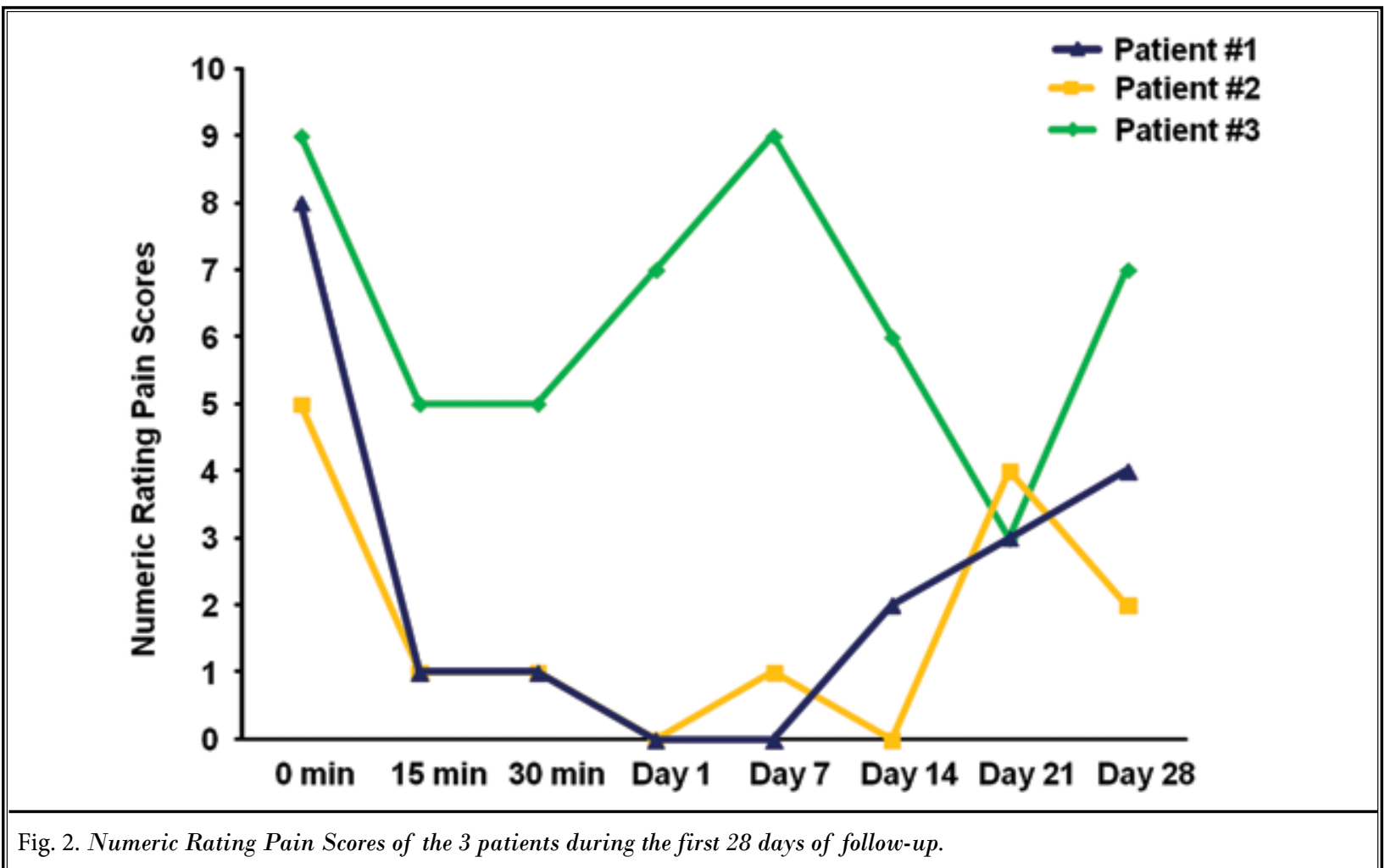


ger pain relief after each subsequent procedure. At one year, she stated she required no additional medication or therapy for her headaches.

\section{Patient \# 3}

A 15-year-old girl presented to the pain clinic with a 2-year history of daily bilateral supraorbital headaches associated with constant pain rated in the moderate-tosevere categories. Her past medical history was significant for periorbital herpetic lesions with a positive PCR for Herpes Simplex Virus since 6 years of age, and facial eczema from the age of 10 months. The headaches were constant and throbbing, exacerbated by performing school work and by loud noise, and were ameliorated by lying down in a dark room and attempting to sleep. Physical examination was unremarkable. The patient was initially diagnosed with chronic migraine headache and Type 1 Chiari malformation. Pharmacotherapy was attempted for a period of 12 months which resulted in no improvement of symptoms. Prescribed agents included valacyclovir, topical acyclovir, Depo-Medrol, gabapentin, escitalopram, trazodone, and a lidocaine/ tetracaine patch. The patient was ultimately referred to neurosurgery who recommended decompression and duraplasty for the Chiari malformation. Although a $50 \%$ relief of headaches was predicted, her headache intensity actually increased following surgery. There was also no change in periorbital herpetic outbreak presentation. She was not able to attend school secondary to debilitating headaches. Brain magnetic resonance imaging (MRI) and lumbar puncture revealed no abnormalities or HSV infection. The patient was finally referred to the pain clinic where SPGB using the Tx360 device was performed. The initial pain level prior to treatment was $9 / 10$ both at rest and with activity. At the minute 15 post-procedure assessment, the pain level had decreased to $5 / 10$ (Fig. 2). However, at the 7 Day follow-up, pain levels returned to baseline, and the procedure was repeated, resulting in longer duration pain relief. On the 28 Day follow-up, her pain level was 7/10. The patient graded her PGIC as "much improved" during the 28-day follow up. Her satisfaction was in the range $3-5$ on the 5 -point scale during the month follow-up. The patient received 9 additional SPGBs within the one year follow-up, each of them providing between 3 weeks to 2 months of pain relief. When evaluated at the one-year follow-up, she stated that her pain was consistently in the "mild" category, typically rated between $2-3 / 10$, and no additional therapy or medication was required. Additionally, she had re- turned to school and had been able to complete her high school requirements towards graduation.

\section{Pain Scores}

All patients demonstrated clinically significant decreases in pain levels at 15 and 30 minutes post-procedure. Two of the 3 patients reported very low levels of pain throughout the follow-up period until Days 21 and 28 when a slight decrease in pain control was noted. The remaining patient (\#3) demonstrated excellent pain relief initially but experienced a recurrence of pre-treatment level pain at the Day 7 follow-up, which was why this patient received the additional SPG block by using the same procedure within the first 28 days. All patients demonstrated substantial pain relief in the initial post-treatment period, while appearing to be unable to maintain the highest levels of pain relief for the full 28-day period. At the 28 Day follow-up, all patients still demonstrated significant pain relief relative to pre-treatment pain levels. None of the patients failed treatment, and all went on to sustainable levels of pain that were managed conservatively without prescription medications or additional interventional therapies.

\section{Pain Inventory}

Pain inventory metrics dropped to a minimum within the first post-procedure day and peaked again within the second week. At the end of 4 weeks all items had increased as compared to the lowest values noted on the first day, but all were still below the baseline (Figs. 3 and 4), except for pain interference with walking ability, which was rated low (1/10) on average, at baseline, and at final evaluations.

\section{Discussion}

Head and face pain can be debilitating conditions affecting millions of people worldwide at a cost of many billions of dollars per year. The extent of the burden of these painful conditions on individuals, families, employers, health care systems, and society as a whole cannot be understated. The cost of migraine alone may be as much as $\$ 19.6$ billion per year in the United States (11) and $\$ 27$ billion in Europe (12). As many as one in 5 of those with chronic migraine and one in 8 of migraineurs with high headache frequency are employable but are not actively gainfully employed (13). Conditions such as these impose a variety of significant burdens. Personal burdens include restrictions placed upon physical, emotional, occupational, academic, social, leisure, and family systems. Societal burdens 


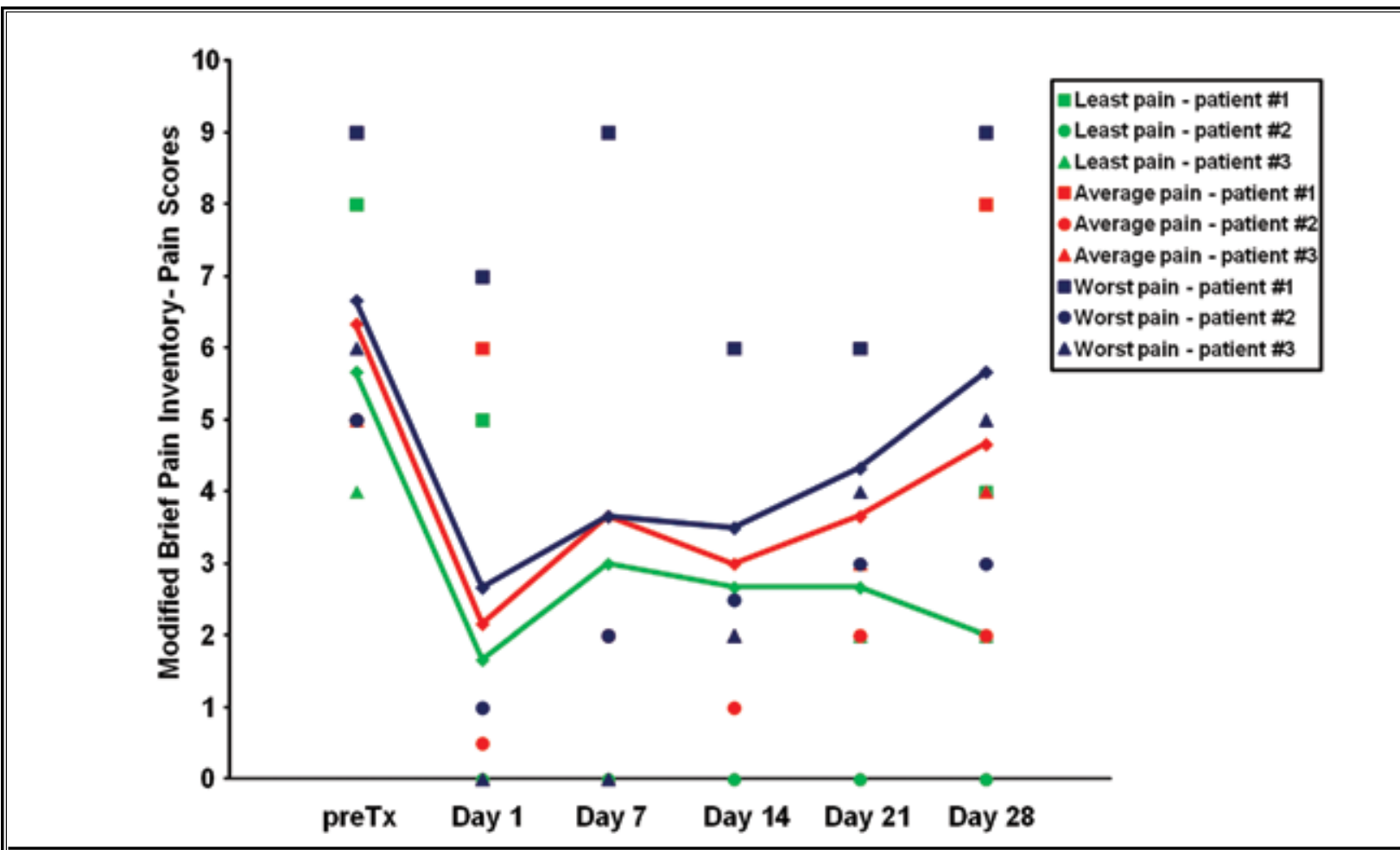

Fig. 3. Modified Brief Inventory - The pain scores (worst, least, and average) of the 3 patients during the first 28 days of follow-up.

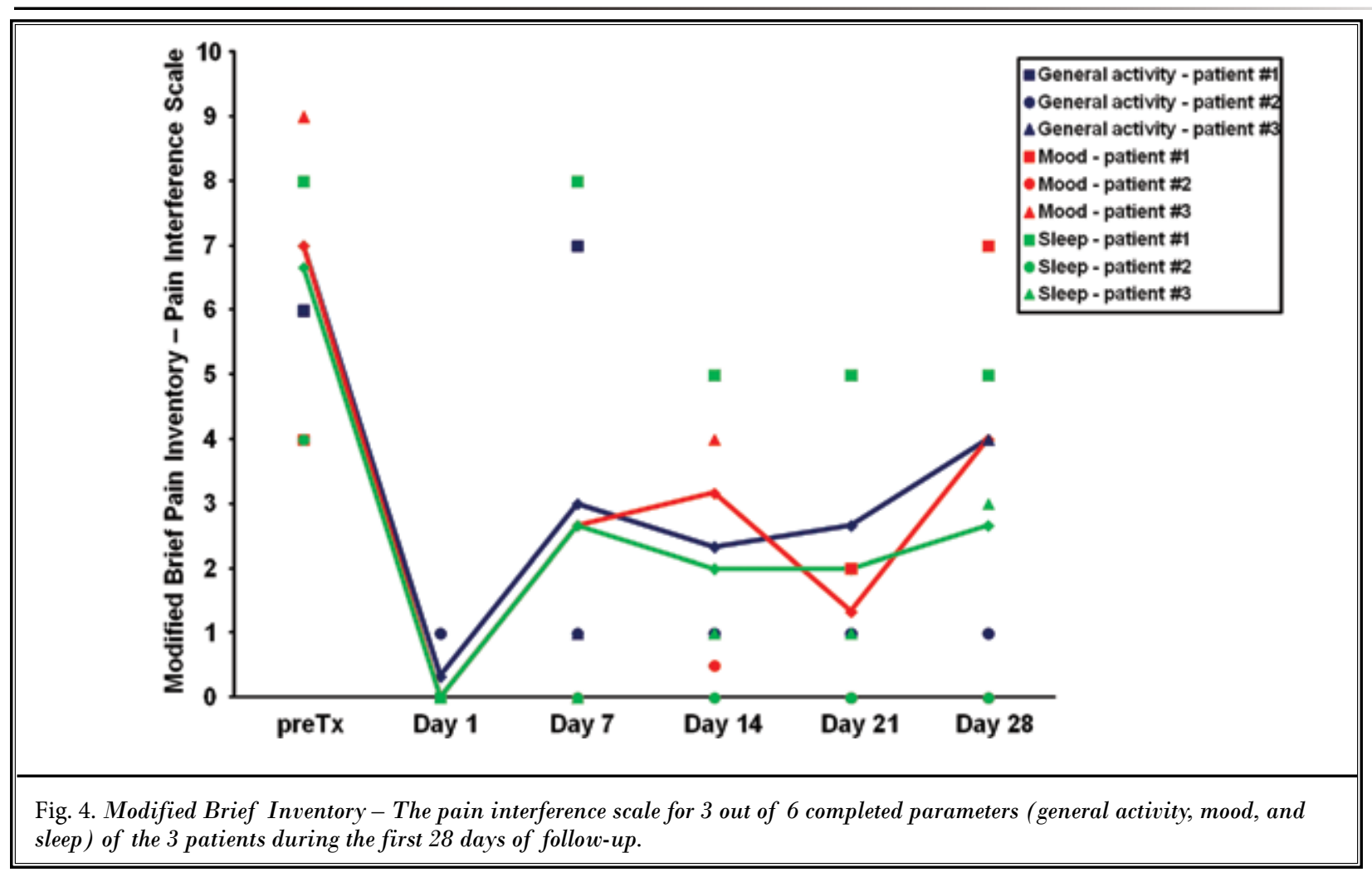


include those direct and indirect costs which are levied against employers and health care systems. These encumbrances must be borne by society as a whole as a result of the pass-through nature of loss from employers to customers and health care systems to taxpayers (14). Unfortunately, the United States health care system is fragmentary at best, wherein many do not have insurance coverage, a regular source of health care, or limited or difficult access to primary care, not to mention an even greater degree of difficulty accessing specialty care (15). The extent of head and face pain impact may indeed continue to increase considering the less than clear picture of the future of health care in this country. The time is optimal to reconsider the status quo in head and face pain treatment.

SPG blocking procedures have various degrees of efficacy. Regardless of the approach, the intent of an SPG intervention is to block or modulate ganglionic function, thereby reducing headache or facial pain. This effect can either be temporary or permanent depending on the technique and utilization of chemical agents, including local anesthetics and neurolytics. Arguably the most aggressive approach is the removal of SPG cells, known as sphenopalatine ganglioneurectomy (16). Other aggressive, invasive methods are the neurosurgical removal of migraine trigger sites in the frontal, temporal, and occipital regions with removal of muscles and/or nerves associated with these areas (17). Stereotactic radiosurgical techniques employ focused irradiation of the pterygopalatine fossa contents with the goal of decreasing aberrant neuronal firing without complete destruction of the SPG (18). Application of Cyber Knife and Gamma Knife systems, the Novalis Tx radiosurgery platform, the Trilogy linear accelerator, and Tomotherapy have also been described.

Interrupting SPG function may also be accomplished with a variety of pharmaceutical agents. Common agents include lidocaine, bupivacaine, cocaine, phenol (in dilute, relatively non-neurolytic concentrations < 5\%), alcohol, and depot steroids (19). Beyond pharmacological and surgical interventions, other alternatives include percutaneous neurostimulation techniques (20). SPG function can also be modulated by electrical stimulation wherein the SPG is stimulated with electrical pulses from a physician controlled external neurostimulator for a period of up to several minutes. A permanently implanted SPG stimulator is also an option used to decrease pain and has been described using infrazygomatic transcoronoid approaches (21). Several different radiofrequency (RF) ablative techniques have been described. Lesser or non-ablative RF treatment using a pulsed-mode may be beneficial as it is associated with a lower degree of tissue destruction (22). Classic RF ablation or RF thermocoagulation is performed up to $80^{\circ} \mathrm{C}$ compared to $42^{\circ} \mathrm{C}$ with the less destructive electromagnetic field-pulsed RF ablation technique (23).

SPG block has been utilized sporadically to manage head and neck related pain since first described by Dr. Greenfield Sluder in 1908 - 1909 (24,25). The traditional procedure requires the patient to be placed supine with the head extended. A cotton-tipped applicator soaked in anesthetic solution is then introduced through the naris until the posterior nasal cavity is approximated. The patients remain in this position in some instances up to 30 minutes while additional anesthetic can be dripped down the shaft of the applicator. The device presented in this manuscript targets an anesthetic agent specifically to the area of mucosa most closely associated with the SPG. This newly revised technique overcomes certain anatomical obstacles related to the classic approach. The primary obstacle is the lack of an easy, direct, "line-of-sight" access to the SPG through the naris. Historically, text book illustrations of the classic transnasal topical technique have erroneously suggested the tip of the cotton swab can somehow be directly approximated to the target mucosa from an anterior-to-posterior trajectory, which obviates the fact that the ganglion is situated more laterally, superiorly, and anterior to the cotton tip once the inferior turbinate is cleared. With the Tx360, the anesthetic is delivered to a site corresponding to actual cadaveric dissections of the location of the SPG, taking into account the variability of precise SPF locations within the population. The flexible catheter tipped device curves around and clears the inferior turbinate when advanced. When the syringe is rotated to its lateral limited position, the anesthetic spray is directed back anteriorly, superiorly, and laterally, directly at the small area of mucosa at the posterior aspect of the middle turbinate with enough diffusion of the spray stream to account for SPF location variability (Fig. 1). The lack of direct access with the rigid, vector-like, classic approach may be an explanation of variable outcomes following SPG block that have been reported over the past century.

The SPG possesses several unique characteristics that render it favorable as a target for treatment of many head and facial pain conditions (1). One of the most important characteristics is the absence of a bony boundary between the nasal cavity and the SPG via 
the SPF. Associated with this strategic communicating foramen lies a thin slice $(1-2 \mathrm{~mm})$ of mucosa (26). This makes infiltration of an anesthetic agent into the pterygopalatine fossa easy to facilitate if accurately delivered. Anatomical research has provided great detail regarding the pterygopalatine fossa and its contents (27-29). Current research has dramatically advanced a thorough understanding of the variability of the SPF topographical location. This understanding is essential to achieve the best possible outcomes with any SPGdirected blocking device. The degree of variability to this critical SPG access point has been documented only relatively recently. The most common location of the SPF was found to be in the area between the middle and superior meatus in approximately $56 \%$ of specimens (27). Topographically, this location corresponds to the lateral insertion of the posterior aspect of the middle turbinate which is also the anterior middle aspect of the SPF. The superior meatus orifice marks the superior aspect of the SPF and is the next most common location found in about $37 \%$ of cases (27). This makes that same posterior middle turbinate tail the inferior anterior aspect of the SPF. Finally, the foramen was shown to be at the middle meatus in several cadaveric dissections and no specimens demonstrated the SPF exclusively located about the superior meatus. Accessory foramina were identified in about $50 \%$ of cases most commonly positioned just below the middle meatus (27). These anatomical considerations may lend themselves to a higher degree of SPG infiltration and subsequent positive outcomes with the featured SPG intervention. The mean size of the SPF was found to be $6.8 \pm 3 \mathrm{~mm}$ vertically and $7.5 \pm 3 \mathrm{~mm}$ in the anteriorposterior direction (27).

An abundance of pathophysiological models for head pain and facial pain exist in the literature. Doubtless, this is a highly complex and controversial area of ongoing investigation. One proposed mechanism that may help to explain the positive results seen with the Tx360 includes modulation of autonomic nerves; a "neurophysiologic rebooting" of sorts. Autonomic and nociceptive projections associated with the SPG innervate supratentorial structures such as blood vessels, pia, and dura and are the components of the trigeminovascular system. Although less well represented in the literature, the existence of a similar extracranial system referred to as the pterygopalatine trigeminovascular system is undeniable. The importance of modulating these systems cannot be underestimated when considering treatment options for a variety of neurovascular orofacial pain syndromes (28). Considering head pain and facial pain from the standpoint of autonomic dysregulation makes the SPG block a reasonable primary treatment option. It may be appropriate to add central or trigeminal autonomic dysregulation to the working diagnoses along with migraine and other painful head and facial disorders. There is also an application for this intervention with patients suffering from the pain associated with head and face cancers (4), and the use of this device can assure more precise delivery of analgesic substances in patients with advanced cancers.

\section{Conclusion}

Results from these 3 patients showed that episodic SPG blocks have an excellent abortive effect. However, repetitive blocking over period of time may prove to break the pain cycle more completely and thoroughly by effecting a longer-lasting modulation of autonomic pathways. This may provide longer periods of pain relief or even in some cases complete resolution, for chronic symptomatology. This is conceptually analogous to the findings noted with long-term dorsal column stimulation used to deregulate neural transmission in complex regional pain syndrome. The ultimate goal is to reduce daily medication consumption, diminish absenteeism and "presenteeism" (30), and decrease emergency department and other unnecessary specialty care visits. All this ultimately allows for increased productivity, improved quality of life, and decreased overall burden to society.

The ease of use of the Tx360 was another important highlight that should allow for broad potential use beyond the pain specialist encounter, even in the busiest of primary care and emergency department settings. SPG block with the Tx360 provides for a fast, inexpensive, easy-to-use intervention in a variety of clinical settings and environments. Future valuable randomized controlled study designs might include direct comparisons to traditional techniques, such as the standard cotton swab technique; abortive efficacy; and longer-term modulation in more chronic scenarios. Another possible study design in light of continuing emphasis in health care cost-control measures might include a comparison between patient-delivered and provider-delivered interventions in areas where local anesthetics are logistically available for home use.

\section{Author Affiliations}

Dr. Candido is Chairman and Clinical Professor of Anesthesiology, Department of Anesthesiology, Advo- 
cate Illinois Masonic Medical Center, Chicago, IL; and the Department of Anesthesiology, University of Illinois, Chicago, IL.

Dr. Massey is a Clinical Research Assistant, Department of Anesthesiology, Advocate Illinois Masonic Medical Center, Chicago, IL.

Dr. Sauer is a Clinical Research Assistant, Department of Anesthesiology, Advocate Illinois Masonic
Medical Center, Chicago, IL.

Dr. Darabad, is a resident, Department of Anesthesiology, Advocate Illinois Masonic Medical Center, Chicago, IL.

Dr. Knezevic is Director of Anesthesiology Research and Clinical Assistant Professor of Anesthesiology, Department of Anesthesiology, Advocate Illinois Masonic Medical Center, Chicago, IL; and the Department of Anesthesiology, University of Illinois, Chicago, IL.

\section{References}

1. Piagkou M, Demesticha T, Troupis T, Vlasis K, Skandalakis P, Makri A, Mazarakis A, Lappas D, Piagkos G, Johnson EO. The pterygopalatine ganglion and its role in various pain syndromes: From anatomy to clinical practice. Pain Pract 2011; 12:399-412.

2. Stechison MT, Brogan M. Transfacial transpterygomaxillary access to foramen rotundum, sphenopalatine ganglion, and the maxillary nerve in the management of atypical facial pain. Skull Base Surg 1994; 4:15-20.

3. Chen Z, Zhao Z, Li M, Yang Y. Clinical significance of trigeminal neuralgia treated using radiofrequency thermocoagulation (RFT) with different approaches. Hua Xi Kou Qiang Yi Xue Za Zhi 2001; 19:240-242.

4. Varghese BT, Koshy RC, Sebastian P, Joseph E. Combined sphenopalatine ganglion and mandibular nerve, neurolytic block for pain due to advanced head and neck cancer. Palliat Med 2002; 16:447-448.

5. Windsor RE, Jahnke S. Sphenopalatine ganglion blockade: $A$ review and proposed modification of the transnasal technique. Pain Physician 2004; 7:283-286.

6. Yang | Y, Oraee S. A novel approach to transnasal sphenopalatine ganglion injection. Pain Physician 2006; 9:131-134.

7. Yarnitsky D, Goor-Aryeh I, Bajwa ZH, Ransil BI, Cutrer FM, Sottile A, Burstein R. 2003 Wolff Award: Possible parasympathetic contributions to peripheral and central sensitization during migraine. Headache 2003; 43:704-714.

8. Maizels M, Geiger AM. Intranasal lidocaine for migraine: A randomized trial and open-label follow-up. Headache 1999; 39:543-551.

9. Bonica JJ (ed). The Management of Pain; with Special Emphasis on the Use of Analgesic Block in Diagnosis, Prognosis, and Therapy. Lea \& Febinger, Philadelphia, PA, 1953.

10. International Headache Society. The International Classification of Headache Disorders: 2nd edition. Cephalalgia 2004; 24 Suppl 1:9-160.

11. Stewart WF, Ricci JA, Chee E, Morganstein $\mathrm{D}$, Lipton $\mathrm{R}$. Lost productive time and cost due to common pain conditions in the US workforce. JAMA 2003; 290:2443-2454.

12. Andlin-Sobocki P, Jonsson B, Wittchen $\mathrm{HU}$, Olesen J. Cost of disorders of the brain in Europe. Eur J Neurol 2005; 12 Suppl 1:1-27.

13. Stewart WF, Wood GC, Manack A, Varon SF, Buse DC, Lipton RB. Employment and work impact of chronic migraine and episodic migraine. J Occup Environ Med 2010; 52:8-14.

14. Buse D, Manack A, Serrano D, Reed M Varon S, Turkel C, Lipton R. Headache impact of chronic and episodic migraine: results from the American Migraine Prevalence and Prevention study. Headache 2012; 52:3-17.

15. The Medicaid Access Study Group. Access of Medicaid recipients to outpatient care. N EngJ Med 1994; 330:1426-1430.

16. Cepero R, Miller RH, Bressler KL. Longterm results of sphenopalatine ganglioneurectomy for facial pain. Am J Otolaryngol 1987; 8:171-174.

17. Guyuron B, Reed D, Kriegler JS, Davis J, Pashmini N, Amini S. A placebo-controlled surgical trial of the treatment of migraine headaches. Plast Reconstr Surg 2009; 124:461-468.

18. Pollock BE, Kondziolka D. Stereotactic radiosurgical treatment of sphenopalatine neuralgia. Case report. J Neurosurg
1997; 87:450-453.

19. Puig CM, Driscoll CL, Kern EB. Sluder's sphenopalatine ganglion neuralgia-treatment with $88 \%$ phenol. Am J Rhinol 1998; 12:113-118.

20. Gronseth G, Cruccu G, Alksne J, Argoff C, Brainin M, Burchiel K, Nurmikko T, Zakrzewska JM. Practice parameter: The diagnostic evaluation and treatment of trigeminal neuralgia (an evidence-based review): Report of the Quality Standards Subcommittee of the American Academy of Neurology and the European Federation of Neurological Societies. Neurology 2008; 71:1183-1190.

21. Tepper SJ, Rezai A, Narouze S, Steiner C, Mohajer P, Ansarinia M. Acute treatment of intractable migraine with sphenopalatine ganglion electrical stimulation. Headache 2009; 49:983-989.

22. Shah RV, Racz GB. Long-term relief of posttraumatic headache by sphenopalatine ganglion pulsed radiofrequency lesioning: A case report. Arch Phys Med Rehabil 2004; 85:1013-1016.

23. Day M. Sphenopalatine ganglion analgesia. Curr Rev Pain 1999; 3:342-347.

24. Farrar JT, Young JP, Jr., LaMoreaux L, Werth JL, Poole RM. Clinical importance of changes in chronic pain intensity measured on an 11-point numerical pain rating scale. Pain 2001; 94:149-158.

25. Mendoza T, Mayne T, Rublee D, Cleeland C. Reliability and validity of a modified Brief Pain Inventory short form in patients with osteoarthritis. Eur J Pain 2006; 10:353-361.

26. Felisati G, Arnone F, Lozza P, Leone M, Curone M, Bussone G. Sphenopalatine endoscopic ganglion block: A revision of a traditional technique for cluster headache. Laryngoscope 2006; 116:1447-1450.

27. Herrera Tolosana S, Fernandez Liesa R, Escolar Castellon Jde D, Perez Delgado 
L, Lisbona Alquezar MP, Tejero-Garces Galve G, Guallar Larpa M, Ortiz Garcia A. [Sphenopalatinum foramen: An anatomical study]. Acta Otorrinolaringol Esp 2011; 62:274-278.

28. Rusu MC. Microanatomy of the neural scaffold of the pterygopalatine fossa in humans: Trigeminovascular projections and trigeminal-autonomic plexuses. Folia Morphol (Warsz) 2010; 69:84-91.

29. Stojcev Stajcic L, Gacic B, Popovic N, Stajcic Z. Anatomical study of the pterygopalatine fossa pertinent to the maxillary nerve block at the foramen rotun- dum. Int J Oral Maxillofac Surg 2010; 39:493-496.

30. Stewart WF, Wood GC, Bruce C, Buse DC, Runken MC, Lipton RB. Longitudinal change in migraine headache-days and indirect cost consequences. J Occup Environ Med 2011; 53:478-487. 